\section{Coronectomy - oral surgery's answer to modern day conservative dentistry}

\author{
V. Patel, ${ }^{1}$ S. Moore ${ }^{2}$ and C. Sproat ${ }^{3}$
}

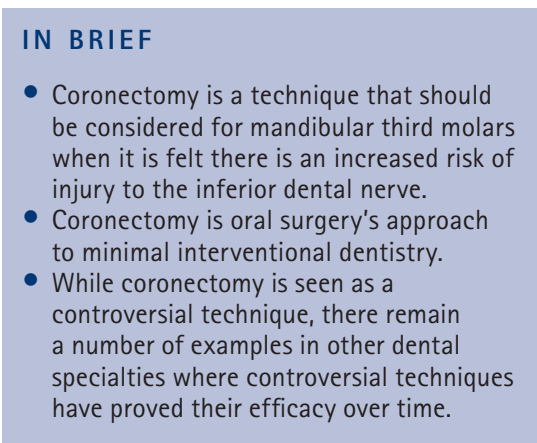

Removal of mandibular third molars is a common oral surgery procedure which is associated with a significant risk of injury to the inferior dental nerve (IDN). In an era of conservative dentistry the technique of coronectomy, which is conservative in terms of surgery and successful in minimising the incidence of IDN injury, has been met with some resistance and has been deemed non-ideal and controversial by many oral surgeons. This article outlines the benefits of coronectomy and highlights some examples from other dental specialities that have embraced conservative principles, despite their detractors.

\section{INTRODUCTION}

Modern dentistry is based on conservative thinking. It appears 'less is more' and most dental specialties have taken this concept on board and applied it to clinical practice. However, this approach may be distant from the textbook theories but in reality provide a range of benefits when we consider both the patient and their teeth.

Restorative dentistry has been at the forefront of a conservative approach to dentistry, largely due to the vast improvements in materials such as composites, allowing the practice of adhesive dentistry. The purpose is to be 'minimal' causing the least amount of trauma to the tooth and the patient, with an overall reduction in post-operative complications and increased long-term success.

As other dental specialties make progress in their conservative approach can oral surgery follow this trend? Coronectomy is a conservative oral surgical procedure which does follow this trend.

The purpose of this article is to highlight the rational for coronectomy and explain how it fits into the conservative

${ }_{1 * 3} \mathrm{Oral}$ and Maxillofacial Surgery, Oral and Maxillofacial Department, Guys Hospital, Floor 23, Great Maze Pond, London, SE1 9RT; ${ }^{2}$ Restorative Dentistry, Guys Hospital, Floor 26, Great Maze Pond, London, SE1 9RT

${ }^{*}$ Correspondence to: Dr Vinod Patel

Email:vinod.patel@ hotmail.co.uk

\section{Refereed Paper}

Accepted 29 April 2010

DOI: $10.1038 /$ sj.bdj.2010.673

${ }^{\circ}$ British Dental Journal 2010; 209: 111-114

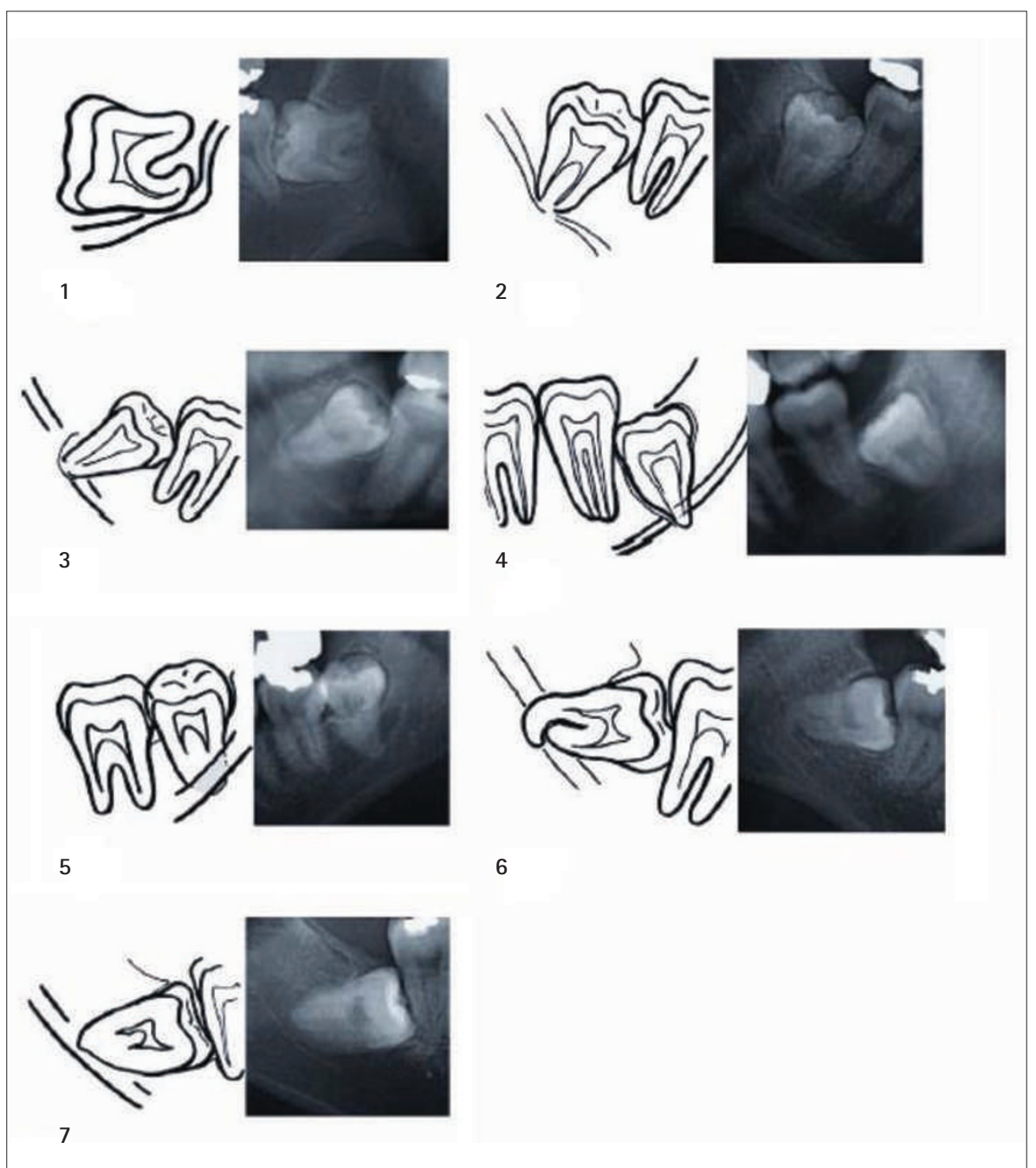

Fig. 1 Radiographic signs of increased risk of inferior alveolar nerve injury: 1, deviation of the canal; 2, narrowing of the canal; 3, periapical radiolucent area; 4 , narrowing of root; 5 , darkening of roots; 6, curving of root; and 7, loss of lamina dura of canal. (Reprinted from Journal of Oral and Maxillofacial Surgery, volume 67, Y. Hatano, K. Kurita, Y.Kuroiwa, H. Yuasa \&t E. Ariji, Clinical evaluations of coronectomy (intentional partial odontectomy) for mandibular third molars using dental computed tomography: a case-control study, pages 1806-1814, copyright (2009), with permission from Elsevier) 
approach of modern dentistry. There are two key issues to the debate around this technique. Firstly oral surgeons should consider procedures or techniques that can reduce the risk of injury to the IDN as a treatment option when treatment planning for the removal of a mandibular third molar (MTM). Secondly, clinicians who feel that electively leaving a root in situ is non-ideal should question this, as there are plenty of occasions when roots are left in situ without consequence. There are examples in other dental specialties where perceived non-ideal treatments were carried out under the umbrella of 'conservative dentistry' and are now accepted treatments currently in use. Some of these examples will be discussed.

\section{DISCUSSION}

\section{Mandibular third molar surgery}

Oral surgery procedures commonly involve the removal of MTMs. A significant risk associated with this procedure is temporary or permanent altered sensation to the lower lip, skin of the chin, teeth and gingivae on the operated side caused by iatrogenic injury to the IDN. The incidence of injury to the IDN when removing MTMs varies from $0.41 \%$ to $8.1 \%$ for temporary lack of sensation and $0.014 \%$ to $3.6 \%$ for prolonged signs and symptoms. ${ }^{1}$ In 'high risk' teeth this may reach $20 \%$. The risk of such an event is commonly evaluated from radiographic examination as part of the treatment planning appointment. Studies have shown that on a panoramic radiograph diversion of the inferior dental canal (IDC), darkening and/or root interruption of the white lines of the canal, narrowing of the canal, and deflection of the roots indicated a possible intimate nerve relationship to the tooth..$^{2-5}$ These radiographic signs are shown in Figure $1^{5}$ along with some other accepted permeations. In the prospective study by Rood and Shehab ${ }^{4}$ of 125 teeth, with signs suggesting an increased risk of nerve involvement, 14\% developed a nerve injury. The Howe and Poyton ${ }^{3}$ study also used radiological predictors as stated above, concluded an IDN injury (IDNI) incidence of 35.64\%. Taking this information into consideration pre-operative knowledge of radiological predictors offers the opportunity to alter the extraction technique to minimise risk to the nerve. ${ }^{6}$
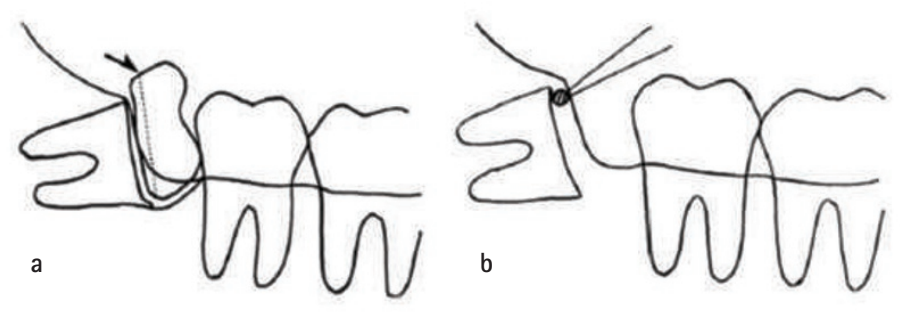

Fig. 2 Coronectomy: A, cutting crown below cement-enamel junction (arrow); $B$, trimming cutting surface to less than 3 to $4 \mathrm{~mm}$ below alveolar crest. (Reprinted from Journal of Oral and Maxillofacial Surgery, volume 67, Y. Hatano, K. Kurita, Y.Kuroiwa, H. Yuasa \& E. Ariji, Clinical evaluations of coronectomy (intentional partial odontectomy) for mandibular third molars using dental computed tomography: a case-control study, pages 1806-1814, copyright (2009), with permission from Elsevier)

\section{Coronectomy}

It is common practice for broken fragments of the root of vital teeth to be left in place and most heal uneventfully. ${ }^{7,8}$ It is on this basis that the concept of coronectomy has evolved. Coronectomy has been defined as a method of removing the crown of a tooth but leaving the roots untouched, which may be intimately related with the inferior alveolar nerve, so that the possibility of nerve injury is reduced (Figs 2-3). . $, 5,9-13^{-13}$ This concept of deliberately removing only the crown of the tooth is known variously as coronectomy, partial root removal, deliberate vital root retention, or partial odontectomy. ${ }^{14}$

Few clinical trials regarding coronectomy have been published, but encouraging results have come from those that have. Renton et al. ${ }^{6}$ and Leung et al. ${ }^{15}$ (randomised clinical trial), Hatano et al. ${ }^{5}$ (case control study) and 0'Riordan ${ }^{1}$ (retrospective study) provided evidence that coronectomy decreases the risk of IDNI when compared to traditional extraction of MTMs. Renton et al. ${ }^{6}$ reported no IDNI in 58 successful coronectomy patients and a 19\% IDNI rate in those having traditional extractions. Leung et al. ${ }^{15}$ showed nine (5\%) patients in the control group presented with IDNI, compared with one $(0.06 \%)$ in the coronectomy group. Hantano et al. ${ }^{5}$ reported that in the extraction group six patients (5\%) suffered IDNI, of which three patients were diagnosed with permanent injury, where as in the coronectomy group one patient (1\%) complained of altered sensation post-operatively which resolved within one month. The retrospective analysis of 0 'Riordan ${ }^{1}$ consisted of 52 patients that underwent coronectomy. In this study there were three cases of transient IDNI which showed resolution one week post

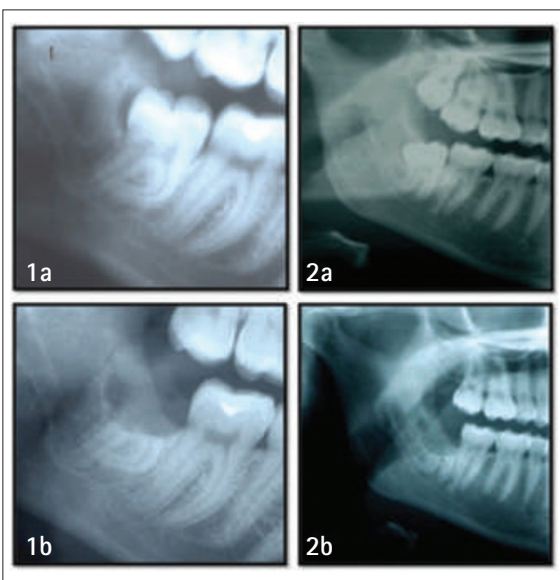

Fig. 3 Radiographic imaging showing pre and post coronectomy of the right mandibular third molar (48)

operatively. One patient developed permanent IDNI, which was thought to be as a result of perforation of the canal due to operator error rather than the coronectomy technique itself. Though the volume of evidence remains small it shows clear trend that coronectomy can reduce IDNI in high risk MTMs.

Coronectomy can be beneficial but success requires both good patient selection and operator technique. There are some simple guidelines which clinicians need to be aware of to avoid failure. They are as follows:

- Teeth with associated infection, particularly infection involving the root portion, should be excluded from this technique ${ }^{16}$

- Teeth that are mobile should be excluded as they act as a mobile foreign body and become a nidus for infection or migration ${ }^{16}$

- There is no evidence for the treatment of the exposed pulp of the tooth and root treatment appears to be contraindicated $^{17-20}$ 
- The technique of leaving the retained root fragment at least $3 \mathrm{~mm}$ inferior to the crest of bone seems appropriate and appears to encourage bone formation over the retained root fragment ${ }^{17-19}$

- Late migration of the root fragment may occur in some cases, but is unpredictable. However, in all cases the root fragments move into a safer position with regard to the nerve, and it can be envisaged that should removal become necessary the nerve would not then be at high risk. ${ }^{16}$ Case reports have suggested that it can take up to ten years for the root fragments to erupt ${ }^{21}$

- The operative site should be primarily closed in a tension free manner ${ }^{16}$

- Dry socket can be treated in the conventional manner with irrigation and dressing. 6

Even though coronectomy has shown evidence of a reduction of IDNI many clinicians are concerned about leaving a large section of root in the mandible. The most common concern is that the retained root may develop a radicular cyst leading to further surgery and morbidity. To date the authors are unaware of any published reports of such an incident following coronectomy. Other concerns relate to post-operative infections. Coronectomy patients also suffer from alveolar osteitis (dry socket) and treatment of these cases remains the same as for traditional extraction alveolar osteitis so long as the root is not mobile. Renton et al. ${ }^{6}$ showed an incidence of 10-12\% for alveolar osteitis in coronectomy patients and treatment included irrigation with chlorohexidine and dressing with Alvogyl ${ }^{\mathrm{TM}}$ (Septodont, France) (butyl aminobenzoate, eugenol, and iodoform) to which all patients responded.

\section{Minimal intervention dentistry}

Just as coronectomy has caused controversy and debate in dentistry there remain examples in other specialties that received a similar reaction. Clinicians have persevered with refining these techniques and evidence has shown their efficacy. Some of these examples are discussed below.

Bleaching of teeth has led to much debate within restorative dentistry. There are two main reasons for this: firstly the legislation surrounding hydrogen peroxide (HP) and secondly the fear of causing tissue damage from its use. Despite the legislation HP is still widely used. All clinicians are aware of the positive use of HP and that it has a valuable role in minimally invasive dentistry. It has been shown that even high concentrations of carbamide peroxide do not alter enamel structure. ${ }^{22}$ The alternative to bleaching teeth with 10\% carbamide peroxide is to resort to destructive 'masking' techniques including porcelain veeners. ${ }^{23}$ Even with controversy around this technique its benefits are obvious and it therefore continues to be used.

Resin-retained bridges (RRB) have been used clinically since the 1970s and offer a more conservative approach to the restoration of edentulous spaces than conventional bridgework. ${ }^{24}$ Over the years techniques have been modified to allow for better retention, life span and aesthetics. Nevertheless, some clinicians have shown some concerns regarding RRBs. Issues raised include that they rely on micromechanical bond and lack retention, the metal wing has potential to allow caries to harbour undetected and they give suboptimal aesthetics. However, their conservative nature in comparison to conventional bridgework minimises long term devitalisation of abutment teeth while still providing the same function. Studies have shown encouraging results for lifespan of a RRB. Audenino et al. ${ }^{25}$ reported the estimated survival probability for the first debonding or failure was $85 \%$ after five years and $71 \%$ after ten years. A meta-analysis ${ }^{26}$ was similar to the five year survival rate of Audenino's group concluding an estimated survival of RRBs of $87.7 \%$ after five years. There has been recent interest in the development of fibre-reinforced resin retained bridges to improve the aesthetics of this type of restoration. However, there are few data supporting longevity. ${ }^{37}$

The atraumatic restorative technique (ART) is a procedure whereby dental caries is excavated manually, eliminating the need for local anaesthesia and use of expensive equipment. The lesion is restored with glass ionomer, an adhesive material that bonds to the tooth structure and releases fluoride as it stimulates remineralisation. ${ }^{27}$ ART can be applied, not only in the dental clinic, but also in institutions for home-bound, physically and mentally handicapped people, in remote areas and in schools. It is considered patient friendly and makes the provision of oral care much easier to patients who are nervous or fearful. ${ }^{28}$ It is used in both restorative and paediatric dentistry. This technique again is far from the traditional approach taught for restoring carious lesions but is a compromise when faced with certain challenging situations. Numerous studies have shown positive results with ART. A meta-analysis of ART $^{29}$ showed mean survival rates for single-surface ART restorations using high-viscosity glass-ionomer in primary dentitions of 95\% after one year to $86 \%$ after three years. The mean survival rates for single-surface ART restorations using high-viscosity glass-ionomer in permanent dentitions were $97 \%$ after one year to $72 \%$ after six years. The simple and effective approach has seen this technique widely accepted worldwide even though as standard routine treatment this procedure would be deemed non-ideal. The World Health Organisation actively promotes atraumatic restorative treatment as a viable approach to meet the need for treatment of dental caries. ${ }^{28}$

The Hall technique, carried out in paediatric dentistry, is arguably one of the most controversial in current use. This is a method of managing carious primary molars effectively with preformed metal crowns, without the use of local anaesthesia, caries removal or tooth preparation of any kind.$^{30}$ The rationale for this treatment was introduced for several reasons. It is known that some children can find the use of local anaesthesia and rotary instruments difficult to accept. ${ }^{31-33}$ The introduction of this technique has not been accepted by all but evidence has shown the technique to be more acceptable to children, parents and GDPs, and to be more effective in preventing pain and sepsis, than conventional restorative techniques. ${ }^{34}$

Oral epithelial dysplasia (OED) is a chronic, often progressive premalignant disorder of the oral mucosa. It is a term used to describe the histopathological changes seen in the oral mucosa and may be graded histologically along a continuum of cellular change as mild, moderate or severe ('carcinoma in situ'). ${ }^{35}$ There is, however, a lack of consensus on the management of oral dysplasia. It is essential 
to remove any possible aetiological factors, and all dysplastic lesions require regular follow-up. ${ }^{36}$ Moderate and severe OED are generally dealt with surgically whereas mild OED tends to be dealt with conservatively. A lesion that has potential to become malignant left in situ could also be interpreted as a controversial and nonideal situation. The conservative management is understandable as removal of lesions would cause loss of oral mucosal tissue mass and could lead to associated morbidity. Furthermore if the aetiological factors are removed including smoking cessation and moderating alcohol intake these lesions may not progress further. ${ }^{38}$

\section{CONCLUSION}

The issue of iatrogenic inferior alveolar nerve damage during the removal of lower third molars continues to be a clinical and medico-legal problem. Coronectomy, a technique which has been shown to reduce the incidence of this, is worthy of exploration ${ }^{16}$ and should be considered as a valid treatment option in high risk cases. Unfortunately there continues to be much resistance to the acceptance of coronectomy in dentistry. The authors agree further research with larger samples and long term follow up would help to support the technique. The short-term results however have shown that this technique is a valid approach in the reduction of IDN injury. The examples stated above had received similar resistance on their introduction into dentistry, however, their continued use in dentistry has proved their worth and the authors feel that this will hold true for coronectomy.

1. O'Riordan B. Uneasy lies the head that wears the crown. Br J Oral Maxillofac Surg 1997; 35: 209.

2. Blaeser B F August M A Donoff R B et al. Panoramic radiographic risk factors for inferior alveolar nerve injury after third molar extraction. J Oral Maxillofac Surg 2003; 61: 417-421.
3. Howe G, Poynton H G. Prevention of damage to the inferior alveolar nerve during the evaluation of mandibular third molars. Br Dent J 1960; 109: 355-363.

4. Rood J P, Shehab B A. The radiological prediction of inferior alveolar nerve injury during third molar surgery. Br J Oral Maxillofac Surg 1990; 28: 20-25.

5. Hatano Y, Kurita K, Kuroiwa Y, Yuasa H, Ariji E. Clinical evaluations of coronectomy (intentional partial odontectomy) for mandibular third molars using dental computed tomography: A case control study. J Oral Maxillofac Surg 2009; 67: 1806-1814.

6. Renton T, Hankins M, Sproat C, McGurk M. A randomised controlled clinical trial to compare the incidence of injury to the inferior alveolar nerve as a result of coronectomy and removal of mandibular third molars. Br J Oral Maxillofac Surg 2005; 43: 7-12

7. Fareed $K$, Khayat $R$, Salins P. Vital root retention, a clinical procedure. J Prosthet Dent 1989; 62: $430-434$

8. Dachi S F, Howell FV. A survey 3,874 routine fullmouth radiographs. Oral Surg Oral Med Oral Patho 1961; 14: 916-924.

9. Knutsson K, Lysell L, Rohlin M. Postoperative status after partial removal of the mandibular third molar. Swed Dent J 1989; 13: 15-22.

10. Freedman G L. Intentional partial odontectomy: report of case. J Oral Maxillofac Surg 1992; 50: 419-421.

11. Freedman G L. Intentional partial odontectomy: review of cases. J Oral Maxillofac Surg 1997; 55: 524-526.

12. Zola M B. Avoiding anesthesia by root retention J Oral Maxillofac Surg 1993: 51: 954

13. Alantar $A$, Roisin-Chausson $M H_{1}$ Commissionat $Y$ et al. Retention of third roots to prevent damage to the inferior alveolar nerve. Oral Surg Oral Med Oral Pathol Oral Radiol Endod 1995; 80: 126

14. Pogrel M A. Coronectomy to prevent damage to the inferior alveolar nerve. Alpha Omegan 2009; 102: 62-67.

15. Leung $Y Y$, Cheung L K. Safety of coronectomy versus excision of wisdom teeth: a randomized controlled trial. Oral Surg Oral Med Oral Pathol Oral Radiol Endod 2009; 108: 821-827.

16. Pogrel M A, Lee J S, Muff D F. Coronectomy: a technique to protect the inferior alveolar nerve. J Oral Maxillofac Surg 2004; 62: 1447-1452.

17. Johnson D L, Kelly J F, Flinton R J et al. Histological evaluation of vital root retention. J Oral Surg 1974 32: 829 .

18. Whitaker D D, Shankle R J. A study of the histologic reaction of submerged root segments. Oral Surg Oral Med Oral Pathol 1974; 37: 919-935.

19. Plata $R$ L, Kelln E E, Linda L. Intentional retention of vital submerged roots in dogs. Oral Surg Oral Med Oral Pathol 1976; 42: 100-108.

20. Cook R T, Hutchens L H, Burkes E J. Periodontal osseous defects associated with vitally submerged roots. J Periodontol 1977; 48: 249-260.

21. Drage N A, Renton T. Inferior alveolar nerve injury related to mandibular third molar surgery: an unusual case presentation. Oral Surg Oral Med Oral Pathol Oral Radiol Endod 2002; 93: 358-361.

22. Kelleher M G. Chemistry and safety of dental bleaching. In: Dental bleaching. UK: Quintessence, 2008.

23. Patel V, Kelleher M, McGurk M. Clinical use of hydrogen peroxide in surgery and dentistry - why is there a safety issue? Br Dent J 2010; 208: 61-64.

24. Tredwin C, Setchell D J, St George G, Weisbloom M. Resin-retained bridges as predictable and successful restorations. Alpha Omegan 2007; 100: 89-96.

25. Audenino G, Giannella G, Morello G M, Ceccarelli $M$. Resin-bonded fixed partial dentures: ten-year follow-up. Int J Prosthodont 2006; 19: 22-23.

26. Pjetursson B E, Tan W C, Tan K, Brägger U et al. A systematic review of the survival and complication rates of resin-bonded bridges after an observation period of at least 5 years. Clin Oral Implants Res 2008; 19: 131-141.

27. Lopez N, Simpser-Rafalin S, Berthold P. Atraumatic restorative treatment for prevention and treatment of caries in an underserved community. Am J Public Health 2005; 95: 1338-1339.

28. Frencken J, van Amerongen $E$, Phantumvanit $P$, Songpaisan Y, Pilot T. Manual for the atraumatic restorative treatment approach to control dental caries. Dental Health International Nederland. http://www.dentaid.org/data/dentaid/downloads/ ART_Manual_English.pdf.

29. van't Hof M A, Frencken J E, van Palenstein Helderman W H, Holmgren C J. The atraumatic restorative treatment (ART) approach for managing dental caries: a meta-analysis. Int Dent J 2006; 56: 345-351.

30. Innes N, Evans D, Hall N. The Hall Technique for managing carious primary molars. Dent Update 2009; 36: 472-478.

31. Rahimtoola $\mathrm{S}$, van Amerongen $\mathrm{E}$, Maher $\mathrm{R}$, Groen $\mathrm{H}$. Pain related to different ways of minimal intervention in the treatment of small caries lesions. ASDC $J$ Dent Child 2000; 67: 123-127.

32. Schriks M C, van Amerongen W E. Atraumatic perspectives of ART: psychological and physiological aspects of treatment with and without rotary instruments. Community Dent Oral Epidemiol 2003 31: 15-20.

33. van Bochove J A, van Amerongen W E. The influence of restorative treatment approaches and the use of local analgesia on children's discomfort. Eur Arch Paediatr Dent 2006; 7: 11-16.

34. Innes N P, Evans D J, Stirrups D R. The Hall Technique; a randomized controlled clinical trial of a novel method of managing carious primary molars in general dental practice: acceptability of the technique and outcomes at 23 months. BMC Oral Health 2007; 7: 18

35. Bánóczy J, Csiba A. Occurrence of epithelial dysplasia in oral leukoplakia. Analysis and follow-up study of 12 cases. Oral Surg Oral Med Oral Pathol 1976. 42: 766-774.

36. Jack $H$, Lee $\mathrm{K}$, Polonowita A. Dilemmas in managing oral dysplasia: a case report and literature review. NZMed J 2009; 122: 89-98.

37. van Heuman C C M, Kreulen C M, Creugers N H J. Clinical studies of fiber-reinforced resin-bonded fixed partial dentures: a systematic review. Eur J Oral Sci 2009; 117: 1-6.

38. Weinberg M A, Estefan D J. Assessing oral malignancies. Am Fam Physician 2002; 65: 1379-1384. 